\title{
Evidence for suppressive mechanisms in attentional selection: Feature singletons produce inhibitory surrounds
}

\author{
JEFFREY R. W. MOUNTS \\ State University of New York, Geneseo, New York
}

\begin{abstract}
Four experiments explored inhibitory mechanisms related to attentional selection. Observers viewed multielement displays and performed a form discrimination task involving a probe element. Also present in the stimulus display was a singleton element (possessing a unique color or orientation). In Experiments 1-3, probe discrimination performance was measured as a function of the distance between the probe and the singleton. Experiment 1 revealed that probe discriminations suffered when the probe was adjacent to the singleton, but improved as the spatial separation between the probe and attentionally salient singleton increased. Experiment 2 added a control condition, revealing that probe discriminations were inhibited near the singleton, but returned to control level performance with increased separation. Further, the amount of inhibition increased with larger stimulus onset asynchronies between the singleton and probe. Experiment 3 demonstrated that the extent of the inhibitory region is spatially mediated. In Experiment 4, the task was modified to one of probe detection. No inhibition was observed in the detection task, indicating that the decrease in probe discrimination performance observed in Experiments 1-3 was not due to observers' inability to detect the probe element.
\end{abstract}

We spend much of our lives in rich visual environments filled with objects of various shapes, sizes, colors, and uses. However, at any given moment in time, we are typically attending to only a small portion of our visual environment. That portion of the environment selected by visual attention enjoys a processing advantage relative to other segments of the environment. This processing advantage for attended items reveals itself in a variety of experimental tasks, either by increasing perceptual sensitivity (e.g., Cheal \& Lyon, 1992; Downing, 1988; Hawkins et al., 1990; Nakayama \& Mackeben, 1989) or speeding detection and discrimination responses (e.g., Eriksen \& Hoffman, 1972; Henderson \& Macquistan, 1993; Posner, Snyder, \& Davidson, 1980).

While the studies cited above suggest that attention acts to facilitate the perceptual processing of attended objects, there is growing evidence that the processing of unattended objects may be inhibited or suppressed (e.g., Caputo \& Guerra, 1998; Cepeda, Cave, Bichot, \& Kim, 1998; Tipper, 1985). Many models of visual selective attention posit that objects compete for control of visual and cognitive processing. It is this competition for processing resources that limits the caliber of the processing of any

This research was supported by grants from the Geneseo Foundation. Portions of this work were presented at the 1998 meeting of ARVO in Ft. Lauderdale, Florida. I thank Jennie Herbert for help in data collection and analysis, and Michele Shady and Robin Tomaselli for useful discussions concerning this work. Correspondence should be addressed to J. R. W. Mounts, Department of Psychology, SUNY Geneseo, 1 College Circle, Geneseo, NY 14454 (e-mail: mounts@geneseo.edu). given object and necessitates selective attention. In these views, selective attention is needed to limit access to higher level visual, cognitive, and/or motor processes (e.g., Allport, 1989; Duncan, 1996; Houghton \& Tipper, 1994; LaBerge \& Brown, 1989) in order to increase the fidelity of these processes.

Several authors have proposed a neural basis for competition among objects and the resulting need for inhibitory processes. For example, Desimone and Duncan (1995; see also Desimone, Wessinger, Thomas, \& Schneider, 1990) pointed out that the size of receptive fields in extrastriate areas are a potential source of problems for the visual system, particularly when multiple objects are in close proximity to one another. Without inhibitory processes, individual neurons (or populations of neurons sharing common receptive field regions) could potentially respond to any and all objects falling within their receptive fields, leading to ambiguity in the coding of features from any single object.

Luck, Girelli, McDermott, and Ford (1997) proposed an inhibition-based model of selective attention that offers a solution to the coding ambiguity problem. Like Desimone and Duncan (1995), Luck, Girelli, et al. argue that the need to inhibit unattended items arises because of the size of receptive fields in extrastriate areas. They contend that this coarse neural coding results in several potential problems for the visual system, such as the accurate coding of features when objects are near one another in the visual field and the possibility of illusory conjunctions (e.g., Prinzmetal, Henderson, \& Ivry, 1995; Treisman \& Schmidt, 1982). The need to resolve these potential cod- 
ing problems forms the basis of Luck, Girelli, et al.'s ambiguity resolution theory. They argue that attentional mechanisms solve the inherent ambiguity in the visual system by suppressing the inputs from unattended items. This eliminates ambiguity at higher levels of the visual system, since the activity of neurons coding information from the region of interest are now determined solely by the attended object. Thus, the theory proposes that inhibiting the processing of objects near the attended object allows the visual system to accurately code - and perhaps bind - the features of the attended object.

An important prediction of ambiguity resolution theory is that attentional selection is required only when objects lie in close proximity to one another (close enough for the objects to fall within the receptive fields of a common population of neurons). Thus, attention need only suppress the processing of unattended objects that are near the attended object; objects farther away from the attended object will be represented by a largely independent population of neurons and should not interfere with the processing of the attended object. In other words, attentional selection should produce a ring of inhibition surrounding the attended item, with the processing of more distant objects being largely unaffected by selective attention mechanisms. This prediction, termed the neighborhood inhibition hypothesis, is the focus of the present study.

Many neurophysiological studies have uncovered effects of attention consistent with this prediction. Within visual processing centers such as areas V4 and IT in the Macaque, attention to an object (or the lack thereof) has been shown to modulate the response rates of individual neurons (e.g., Chelazzi, Miller, Duncan, \& Desimone, 1993; Luck, Chelazzi, Hillyard, \& Desimone, 1997; Moran \& Desimone, 1985; Motter, 1993). While many neurophysiological studies have provided evidence consistent with the neighborhood inhibition hypothesis, to date there is meager support for this hypothesis from behavioral studies. In fact, the results from prevalent experimental paradigms appear to counter the neighborhood inhibition hypothesis. Many studies that have explored the attentional field suggest that it takes the form of a gradient filter, with processing efficiency (or the flow of information) falling monotonically with increased distance from the attentional focus (e.g., Cheal, Lyon, \& Gottlob, 1994; Henderson \& Macquistan, 1993; LaBerge, Carlson, Williams, \& Bunney, 1997). Nonetheless, several recent behavioral studies have reported results consistent with neighborhood inhibition (e.g., Bahcall \& Kowler, 1999; Caputo \& Guerra, 1998; Cave \& Zimmerman, 1997).

One method that appears particularly well suited for the detection of the type of inhibitory processes predicted by neighborhood inhibition is the attentional capture paradigm. Studies examining the phenomenon of attentional capture have found that, given the appropriate perceptual set on the part of the observer (e.g., Bacon \& Egeth, 1994; Folk \& Remington, 1998; Folk, Remington, \& Wright, 1994; Theeuwes, 1992; Yantis \& Egeth, 1999), certain stimulus characteristics (such as abrupt onsets and feature singletons) can capture attention. If a stimulus characteristic does capture attention, we would expect an inhibitory surround to manifest around the attended item, leading to greater performance decrements for targets near an attentionally salient distractor. Unfortunately, most studies in this area have failed to break down the data in terms of distractor-target separation. An exception, Joseph and Optican (1996), examined the time course of attentional capture (by manipulating the distractor-target stimulus onset asynchrony [SOA]) using a uniquely oriented bar among a field of otherwise homogeneous bars. The distractor and target could appear at one of four locations in the display (the vertices of an imaginary square), resulting in two possible distractor-target separations. Although they observed inhibition for both small and large separations, Joseph and Optican did not make a direct comparison of the amount of inhibition between the two separation distances. A visual analysis of their Figure 4 suggests mixed results, with some observers showing greater inhibition with smaller separations, particularly at shorter SOAs. A possible reason for the lack of stronger findings is the size of the target-distractor separations examined-their small separation was approximately $5.5^{\circ}$. As we will see in the present study, stronger inhibitory effects obtain with smaller separations.

Using a modification of the attentional capture paradigm, Caputo and Guerra (1998) observed an inhibitory region surrounding an attentionally salient feature singleton, consistent with the predictions of neighborhood inhibition. In their task, seven items were arrayed along an imaginary circle. The target was a unique shape (diamond among disks or vice versa), while the distractor possessed a unique color. Three target-distractor separations were possible, with the smallest being approximately $3^{\circ}$. Measuring the threshold for detecting an increase in the length of a line presented in the center of the target, Caputo and Guerra found that the line length threshold increased as the target-distractor separation decreased, with the largest threshold increase observed when the distractor and target were adjacent to each other. This suggests that the capture of attention by the color singleton resulted in a ring of inhibition surrounding its location.

Other behavioral studies have revealed results consistent with neighborhood inhibition. Cave and Zimmerman (1997) asked observers to detect the presence of a target letter within an eight-letter array. With practice on this primary task, target detection efficiency increased, yielding nearly flat search slopes. Cave and Zimmerman also included a secondary task: On a portion of the trials, a probe (a small dot) appeared within the target display. Cave and Zimmerman measured observers' simple reaction times to this probe. As search efficiency increased with practice, reaction times to probes presented near the target became elevated relative to probes more distant from the target, while probes presented at the target location were speeded. This pattern too suggests that attention to the target item led to the suppression of the processing of items in the target's vicinity-in this case, slowing reaction time to the probe stimulus. 
Finally, Bahcall and Kowler (1999) asked observers to identify 2 target letters embedded within a circular array of 24 letters. Target displays were were preceded by pretrial displays of 24 alphanumeric characters in the same spatial arrangement. Within these pretrial displays, unique colors or specific character values were used to signify subsequent target locations. Bahcall and Kowler found that letter identification performance decreased as the distance between the target letters became smaller, regardless of the cue used to direct attention. This pattern obtained even when physical cues defining the targets (e.g., color differences) were presented only in the pretrial display. By removing the physical cues prior to the presentation of the target display, Bahcall and Kowler were able to rule out sensory-based explanations for the performance decrement, since the target displays for the various target separations were identical in the color, number, and positioning of the letters.

As noted, a small number of studies have obtained results consistent with the neighborhood inhibition hypothesis. The present study seeks to extend these findings, using a form identification task. Observers' ability to judge the form of a briefly presented probe was used to gauge attentional effects. The focus of attention was manipulated exogenously via a feature singleton-an item that possessed either a unique color or orientation relative to the other items in the stimulus display. Across the set of experiments, probe discrimination ability was measured as a function of its distance from an attentionally salient singleton. The neighborhood inhibition hypothesis predicts that probe discriminability should be poorest near the singleton, with performance improvements as the distance between the probe and singleton increases.

\section{EXPERIMENT 1}

The first experiment was designed to evaluate the neighborhood inhibition hypothesis. A dual-task procedure was used, with observers making discriminations involving the forms of two display elements - specifically, the singleton and the probe. The sequence of trial events is shown Figure 1. At the beginning of each trial, an array of elements - containing one element that was attentionally salient due to a unique color or orientation - was presented to the observers. The probe element was not identifiable in this initial display, but rather was revealed by removing a portion of one of the array elements. ${ }^{1}$ In order to encourage observers to direct attention to the singleton, observers were required to make a discrimination involving the singleton's form. To allow time for attention to be allocated to the singleton, the unveiling of the probe was offset briefly in time from the appearance of the singleton display. The observers were also asked to make a second form discrimination involving the form of this probe element. The observer's probe discrimination performance was measured as a function of the distance between the probe and the singleton. According to neighborhood inhibition, we would expect a decrease in sensitivity when the probe appears adjacent to the singleton, since the processing of the probe would be suppressed. As the distance between the probe and singleton increases, the discrimination sensitivity should return to an asymptotic level of performance.

\section{Method}

Observers. Three observers (including J.M.) participated in this study. The 2 observers other than J.M. were from the Geneseo Community and were paid for their participation. All observers reported normal color vision and normal or corrected-to-normal acuity.

Apparatus. Stimuli were presented on an AppleVision 1710 monitor set to a resolution of $1,152 \times 852$ pixels with a frame rate of $75 \mathrm{~Hz}$. Experimental procedures were controlled by a Power Macintosh 7200 . Observers were seated $57 \mathrm{~cm}$ from the display, with head stabilization aided through the use of a chin and forehead rest. Responses were made via a standard keyboard. All sessions were conducted in a darkened room.

Stimulus display. Stimuli were block 8 s, each subtending a visual angle of $.76^{\circ} \times .45^{\circ}$; the width of each line segment making up the block 8 was $.13^{\circ}$. The display on each trial consisted of an array of 60 elements arranged in a hexagonal grid (minus the center item) with an interelement visual angle of $1.5^{\circ}$ between the centers of neighboring elements. The block $8 \mathrm{~s}$ were either orange $(x=.47, y=$ .45 ; luminance: $\left.11.93 \mathrm{~cd} / \mathrm{m}^{2}\right)$ or green $(x=.35, y=.54$; luminance: $11.89 \mathrm{~cd} / \mathrm{m}^{2}$ ) and presented against a black background. Singleton and probe elements appeared at 1 of 12 locations; the center of each potential singleton/probe location was $3.98^{\circ}$ from the fixation point. Figure 2 contains an example of the stimulus array, with the 12 possible singleton/probe locations highlighted.

Procedure. Observers began each trial by fixating a gray cross (luminance $=12.16 \mathrm{~cd} / \mathrm{m}^{2}$ ) in the center of the screen. Trial initiation was accomplished by pressing a key on the computer keyboard. After a delay of $250 \mathrm{msec}$, the stimulus array appeared. The initial stimulus display consisted of 59 homogeneous elements and the singleton. For Observers J.M. and A.M., all stimulus elements were oriented vertically, and the singleton differed in color-orange among green for half of the experimental sessions and green among orange for the other half. For Observer S.L., all stimulus elements were green, and the singleton differed in terms of orientation- $22^{\circ}$ left of vertical among vertical elements $\left(0^{\circ}\right)$ for half of the sessions, and $0^{\circ}$ among $22^{\circ}$ for the other half. This initial display remained on the screen for $67 \mathrm{msec}$, at which time either the top or bottom line segment was removed from the probe element. This display (containing both the singleton and the probe) remained for $53 \mathrm{msec}$, after which it was replaced by a blank screen including only a fixation point. This sequence of trial events is shown in Figure 1.

On each trial, observers made two responses. The first involved the form of the singleton. On half of the trials, the singleton was missing the center horizontal line segment, yielding a block 0 rather than an 8 . Observers' responses for this discrimination were made using two keys on the computer's number pad (right hand). The second decision involved the form of the probe-specifically, whether it lost its bottom or top segment (yielding a block $A$ or upside-down A). This decision was entered via two keys on the left side of the keyboard (left hand). Both responses were unspeeded, and accuracy was stressed. Observers were instructed to treat the singleton discrimination task as the primary task and to make this response first, followed by the probe response. Errors resulted in a computer beep and a message on the screen indicating the type(s) of error(s) committed.

Observers completed one practice and four experimental sessions. For each observer, the type of singleton (e.g., green among orange or vice versa) alternated between experimental sessions. Each session contained 25 practice followed by 576 experimental trials. Across the 576 trials, the singleton appeared equally often at each of the 12 possible locations, as did the probe. Each possible combination of singleton and probe location occurred four times 


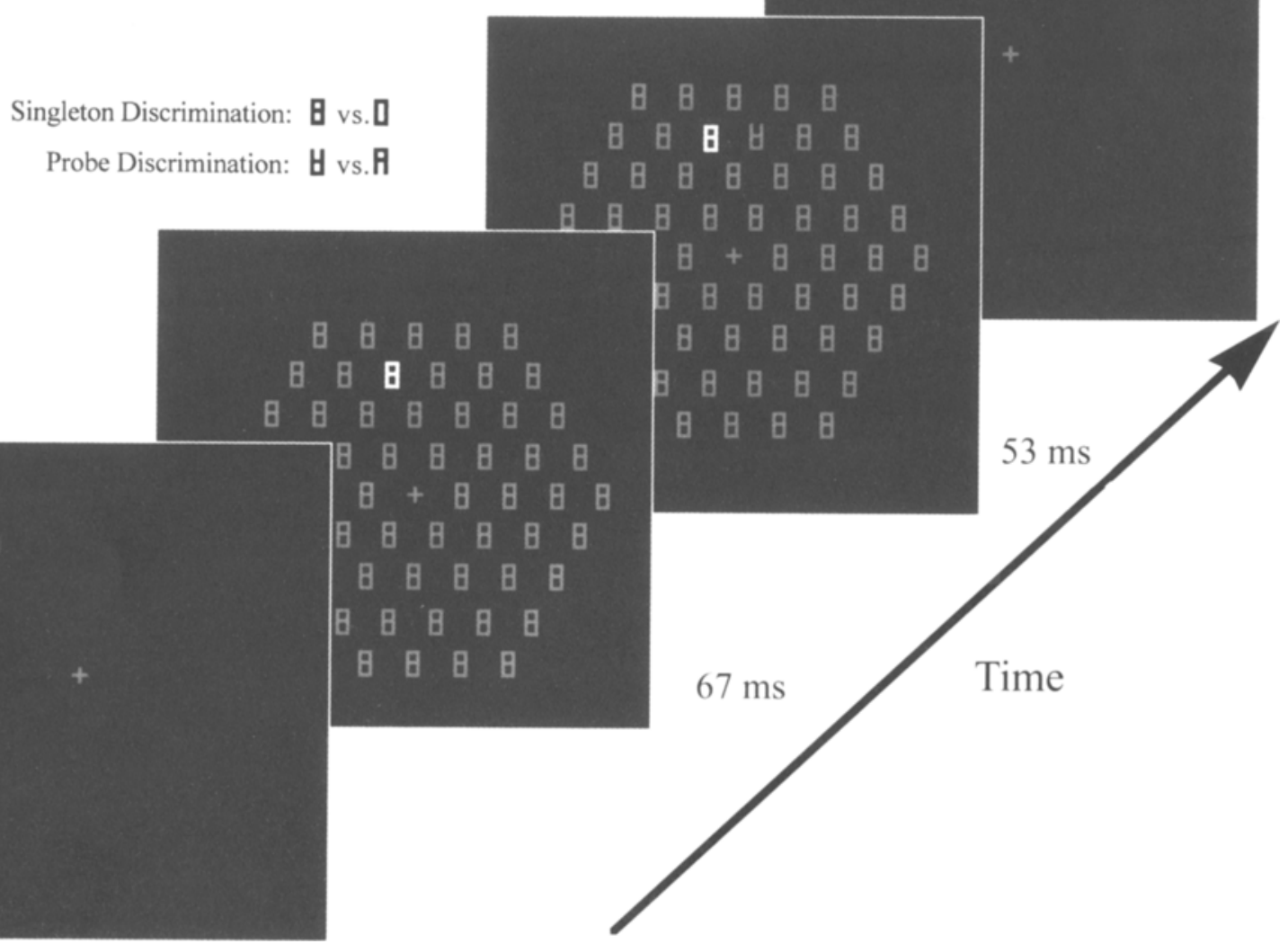

Figure 1. The sequence of events within a trial in Experiment 1. The fixation display is followed by an array of elements that includes the singleton element. Sixty-seven milliseconds later, the probe element is revealed by removing a line segment from one of the display elements. After $53 \mathrm{msec}$, the entire stimulus array is removed and replaced by the fixation display. Observers made two form discriminations, one involving the singleton, the other involving the probe.

(including the singleton and the probe sharing the same location), meaning that the location of the singleton was perfectly uninformative as to the subsequent location of the probe. The four trials for each possible location pairing consisted of each type of singleton $(0$ vs. 8) being paired with each type of probe (A vs. upside-down A). Observers proceeded at their own pace, with a message appearing every 72 trials, indicating that they should take a short rest break. Each session lasted approximately $1 \mathrm{~h}$.

\section{Results and Discussion}

Discrimination accuracy for the singleton form was uniformly high across all 3 observers (J.M., 88\%; A.M., 95\%; S.L., 94\%), suggesting that the observers were directing attention to the singleton. Probe discrimination sensitivity as a function of distance between the singleton and the probe was calculated using all of the trials. The results reported below were changed little by excluding those trials in which an incorrect singleton discrimination was made.

For each singleton-probe distance, the observer's probe discrimination sensitivity $\left(d^{\prime}\right)$ was calculated. ${ }^{2}$ Figure 3 shows probe $d^{\prime}$ s for each of the 3 observers as a function of singleton-probe distance. An analysis of variance (ANOVA) was performed on the session $d$ 's of each individual observer, treating singleton-probe distance ( 10 levels) as a repeated measure. This ANOVA was reliable for all 3 observers [J.M., $F(9,27)=10.91, p<.001$; A.M., $F(9,27)=5.52, p<.001$; S.L., $F(9,27)=3.22, p<.01]$. The neighborhood inhibition hypothesis predicts that probe discrimination sensitivity should increase with distance from the singleton stimulus. Linear contrasts were performed on the accuracy data from each observer (excluding the 0 distance) in order to evaluate this prediction. ${ }^{3}$ This linear contrast was reliable for Observer J.M. $[F(1,3)=68.18, p<.01]$ and Observer A.M. $[F(1,3)=$ $25.13, p<.05]$. Although Figure 3 suggests that this trend may also be present for Observer S.L., this contrast did not approach significance $[F(1,3)=1.48$, n.s. $]$.

The shape of the functions relating probe discrimination sensitivity to distance from the singleton are consistent with the predictions of ambiguity resolution theory. 


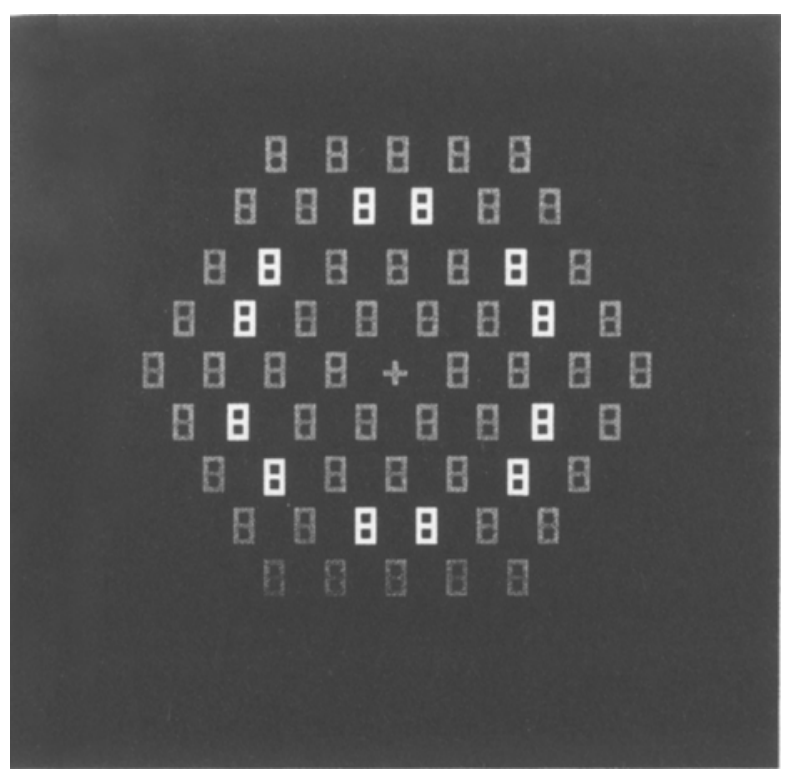

Figure 2. The stimulus array used in Experiment 1. The highlighted elements denote the 12 possible singleton and probe locations.

Processing of elements near the singleton appear to be inhibited, with this inhibition diminishing with increased distance from the singleton. Further, probe discrimination ability appears to asymptote at extreme distances, suggesting little or no impact of the presence of a distant singleton item. However, the design of the present experiment makes it impossible to confirm this observation. This is one of the questions that will be addressed in Experiment 2 .

The inhibition surrounding the singleton (as well as the facilitation of the singleton) appeared to be much greater for Observers J.M. and A.M. Note that for both of these observers, the singleton took the form of a unique color, whereas for Observer S.L., the singleton was defined by a unique orientation. To preview, this difference between color and orientation singletons will appear in the remaining experiments. A likely explanation for this asymmetry is that the color singletons in the present study are more salient, leading to a more robust attentional effect. Nonetheless, orientation singletons produced the same qualitative effects across the series of experiments, suggesting that the observed results are due to general attentional mechanisms, as opposed to feature-specific interactions in the processing of the display elements.

\section{EXPERIMENT 2}

The task demands of Experiment 1 required observers to make a discrimination regarding the singleton; this was done to ensure that attention was directed to this element. However, it is likely that attention would be directed to the singleton, even if a discrimination was not required. For example, Theeuwes (1992) proposed that feature singletons lead to the automatic "capture" of at- tention, regardless of the goals of the observer. However, Bacon and Egeth (1994) found that observers had to be in a "singleton" search mode in order for attention to be captured by an irrelevant singleton. In the present task, observers may have adopted a singleton search mode when searching for the probe. This is because the probe itself was both a form singleton and an offset singleton (i.e., it is the only abrupt offset in the stimulus display and it has a unique form relative to the remainder of the homogeneous items; see note 1). However, the localized inhibition observed in Experiment 1 may not be due to attentional selection. Another possibility is that it is the requirement of an identification of the singleton item that engages the inhibitory mechanisms, and not merely the singletons' attentional salience. In other words, inhibition arose because observers had to respond to two neighboring stimuli (see Bahcall \& Kowler, 1999). In Experiment 2, the method was changed to evaluate this possibility; observers were required to make a discrimination of the probe element only. This change in method yields two advantages: (1) an evaluation of whether an inhibitory region surrounds a singleton when it does not require a response, and (2) eliminating a response to the singleton allowed for the singleton to be withheld on some trials, yielding a true control condition against which facilitation and inhibition could be assessed.

A second change in the method for Experiment 2 involved the SOA between the singleton and the probe stimulus. In Experiment 1, an SOA of $67 \mathrm{msec}$ was used. In Experiment 2, the SOA could assume three different values: 0,40 , and $80 \mathrm{msec}$. Estimates for the allocation time for exogenously cued attention are in the range of 60-100 msec (Cheal et al., 1994; Müller \& Rabbitt, 1989; Nakayama \& Mackeben, 1989). Thus, if the facilitation and inhibition observed in Experiment 1 are attentional in nature, we would expect little facilitation or inhibition at the 0-msec SOA, with the magnitude of these effects increasing at longer SOAs. A final change involved the use of a poststimulus mask to limit the processing time for the stimulus elements.

\section{Method}

Observers. Four observers (including J.M.) participated in this study. The 3 observers other than J.M. were from the Geneseo Community and were paid for their participation. All observers reported normal color vision and normal or corrected-to-normal acuity.

Procedure. The apparatus and stimulus displays were identical to those used in Experiment 1, as were the general procedures. As in Experiment 1, the initial stimulus display consisted of 59 homogeneous elements, plus the singleton. For Observers J.M. and S.W., all elements were vertical, and the singleton differed in color-orange among green for half of the experimental sessions, and green among orange for the other half. For Observers A.B. and M.B., all elements were green, and the singleton differed in terms of orientation $-22^{\circ}$ left of vertical among vertical elements $\left(0^{\circ}\right)$ for half of the sessions, and $0^{\circ}$ among $22^{\circ}$ for the other half. For all observers, a poststimulus mask followed the stimulus display. The mask consisted of 12 line segments randomly arrayed at each of the 60 element locations. The masking segments were the same size as the line segments composing the block $8 \mathrm{~s}$; the orientations were randomly divided among $0^{\circ}, 45^{\circ}, 90^{\circ}$, and $135^{\circ}$; and half of the line segments were green 

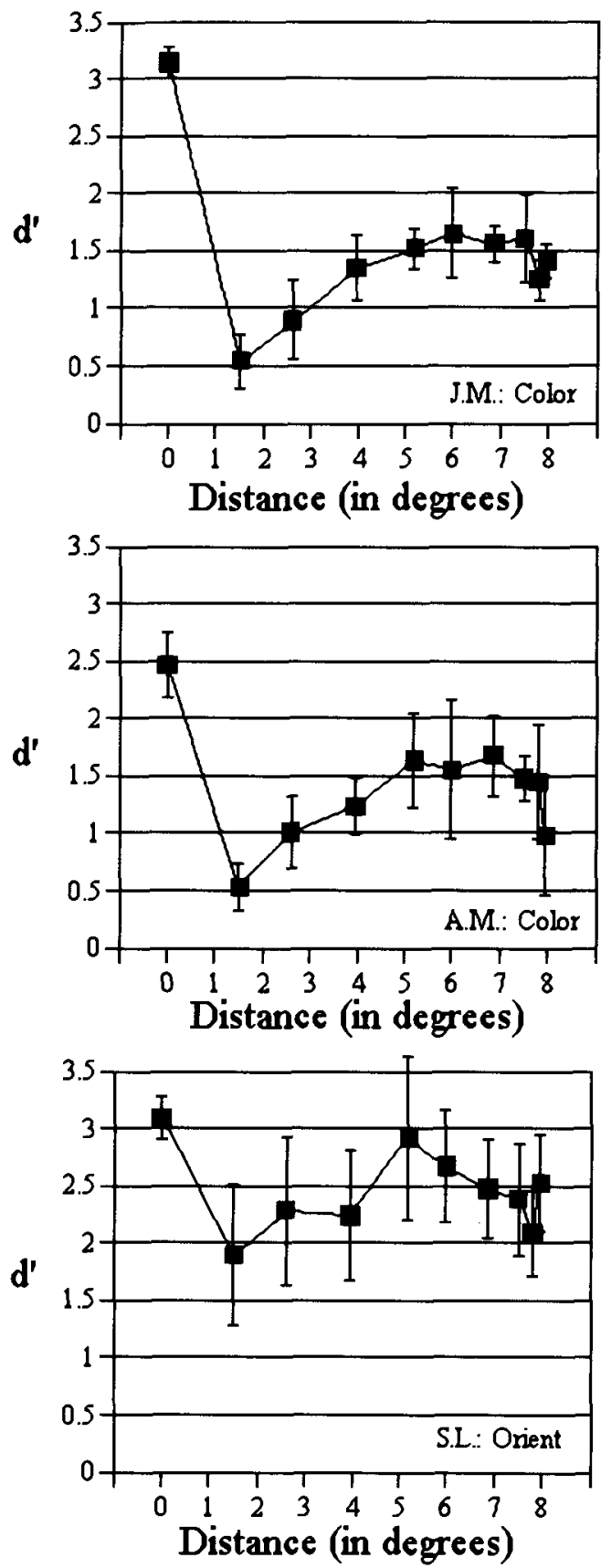

Figure 3. Form discrimination sensitivity $\left(d^{\prime}\right)$ for the 3 observers at each of the 10 singleton-probe separations in Experiment 1 . A singleton-probe separation of 0 indicates that the singleton and probe were the same element. Error bars depict $\pm 1 S E$.

while the other half were orange (the same color values used for the stimulus elements).

Observers completed 25 practice and 936 experimental trials. On 312 of the trials, both the singleton and probe were present in the initial stimulus display ( $0-\mathrm{msec} \mathrm{SOA})$, which remained on the screen for $107 \mathrm{msec}$ before being replaced by the poststimulus mask. On another 312 of the trials, the SOA between the singleton and the probe displays was $40 \mathrm{msec}$, while for the remaining 312 trials, the SOA was $80 \mathrm{msec}$. For both the $40-\mathrm{msec}$ SOA and the $80-\mathrm{msec}$
SOA, the probe display followed the singleton display and remained for $107 \mathrm{msec}$, at which time it was replaced by a poststimulus mask. The sequence of events within a trial is shown in Figure 4. Trials involving the three different SOAs were randomly intermixed within the session. On each trial, observers made a single response regarding the probe, identical to the type of discrimination made in Experiment 1 (top vs. bottom segment missing). Errors resulted in a computer beep.

Observers completed two practice and six experimental sessions. For each observer, the type of singleton (e.g., green among orange or vice versa) alternated between experimental sessions. Across the 936 experimental trials, the probe appeared equally often at each of the 12 possible stimulus locations. The singleton also appeared equally often at each of the 12 possible locations ( 72 trials each) and was absent from the display for an additional 72 trials. As in Experiment 1 , the location of the singleton was perfectly uninformative as to the subsequent location of the probe. Each possible combination of singleton and probe location occurred twice-once for each type of probe stimulus. Observers proceeded at their own pace, with a message appearing every 117 trials indicating that they should take a short rest break. Each session lasted approximately $1 \mathrm{~h}$.

\section{Results and Discussion}

For the various singleton-probe distances at each of the three SOAs, the observer's sensitivity $\left(d^{\prime}\right)$ to make probe discrimination was measured. Figure 5 shows sensitivity relative to the control condition for each combination of singleton-probe distance and SOA. The legend contains the sensitivity for each of the control conditions for each observer. For the 3 observers demonstrating the inhibitory effect (J.M., S.W., and A.B.), sensitivity at the singleton location is enhanced relative to the control condition, while sensitivity falls below that for the control condition at the locations adjacent to the singleton, confirming the presence of both facilitation and inhibition, as suggested by the results of Experiment 1. ANOVAs were conducted on the $d^{\prime}$ data of each observer, with SOA (three levels) and singleton-probe distance (11 levels including the control) treated as repeated measures. For Observer J.M., the main effect of distance was reliable $[F(10,50)=5.42, p \leq .001]$, while the main effect of SOA was not $[F(2,10)=2.15$, n.s.]. The interaction between these two factors approached significance $[F(20,100)=$ $1.61, p=.06]$. The ANOVA on the data of Observer S.W. also revealed a main effect of distance $[F(10,50)=17.78$, $p \leq .001]$, no main effect of SOA $[F(2,10)=2.08$, n.s. $]$, and a marginal interaction between SOA and distance $[F(20,100)=1.56, p=.08]$. For Observer A.B., both the factors of distance $[F(10,50)=5.83, p \leq .001]$ and SOA $[F(2,10)=10.41, p \leq .005]$ proved reliable, while the interaction between these two factors did not $[F(20,100)=$ 1.09 , n.s.]. For Observer M.B., SOA yielded a main effect $[F(2,10)=18.27, p \leq .001]$, while the main effect of distance $[F(10,50)=1.12$, n.s. $]$ and the interaction between SOA and distance $[F(20,100)=.80$, n.s. $]$ did not approach significance.

In order to evaluate whether sensitivity increases with distance from the singleton, linear contrasts identical to those used in Experiment 1 were performed at each SOA for each of the 4 observers. For Observer J.M., only the linear contrast for the SOA-40 condition proved reliable 


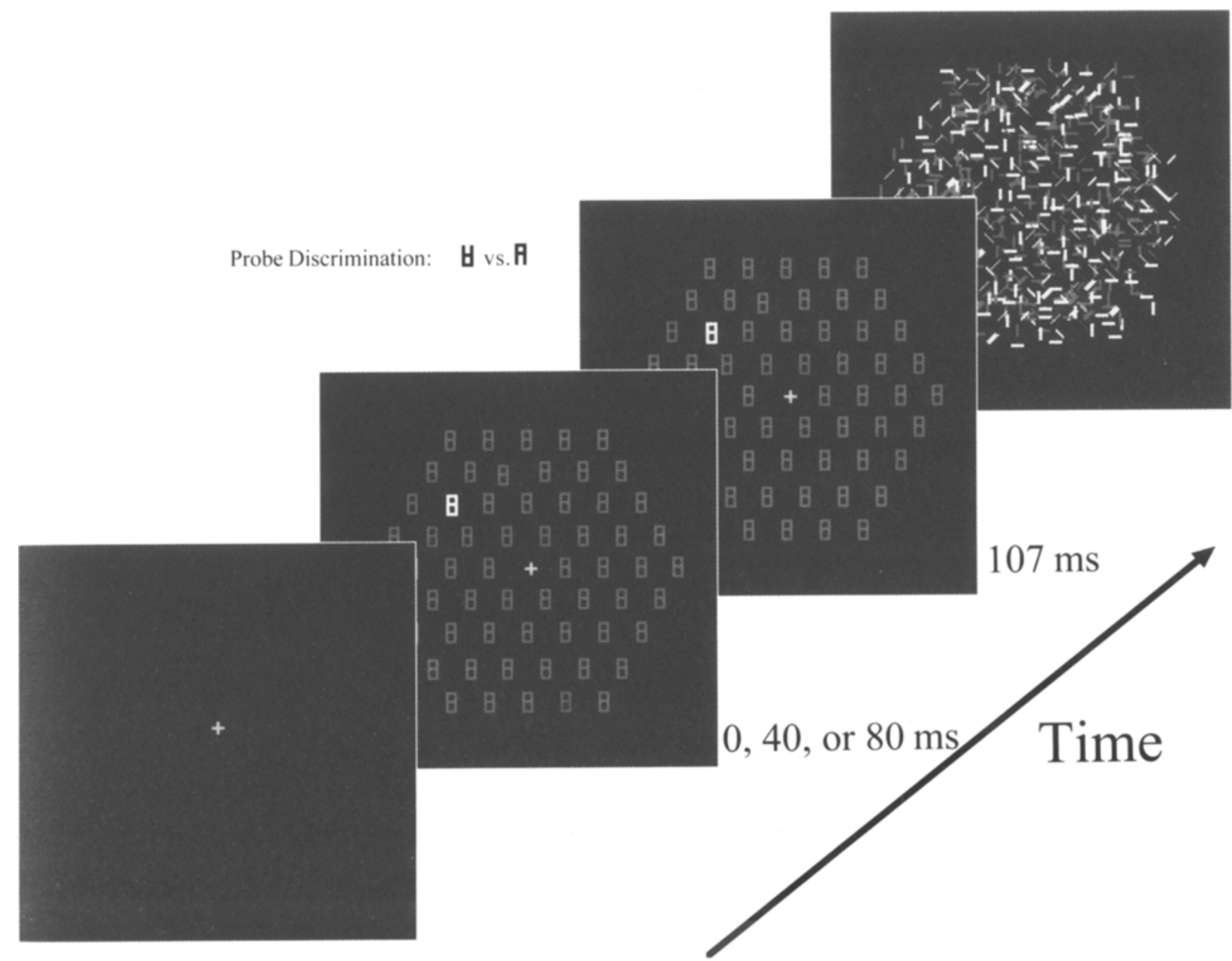

Figure 4. The sequence of events within a trial in Experiment 2. The fixation display is followed by an array of elements that includes the singleton element. On trials with a stimulus onset asynchrony (SOA) of 0 , the probe element was also present in this initial array. On SOA -40 and SOA-80 trials, the probe element is revealed by removing a line segment from one of the display elements after 40 or $80 \mathrm{msec}$, respectively. On all trials, a poststimulus mask replaced the stimulus array $107 \mathrm{msec}$ after the appearance of the probe element. Observers made a single response concerning the form of the probe element.

$[F(1,5)=63.37, p \leq .001]$. For Observer S.W., the linear contrast for SOA 40 approached significance $[F(1,5)=$ $3.45, p=.12$ ], while the linear contrast for SOA 80 was found to be reliable $[F(1,5)=8.21, p \leq .05]$. For Observer A.B., both the SOA $40[F(1,5)=7.79, p \leq .05]$ and SOA $80[F(1,5)=13.73, p \leq .01]$ reached significance. None of the three linear contrasts approached significance for Observer M.B.

The data from 3 of the 4 observers displayed a similar pattern to those obtained in Experiment 1 . The data of the 4th observer (M.B.) failed to show this effect. Several explanations for the pattern of results exhibited by M.B. are viable. First note that Observer M.B. was exposed to orientation singletons, which also produced weaker effects in Experiment 1. Second, and perhaps more important, is the fact that Observer M.B.'s baseline performance is rather low $\left(d^{\prime} \mathrm{s}\right.$ in the control condition range from .55 to
.89). Observer M.B. aside, the data of the other $3 \mathrm{ob}-$ servers show the same pattern as that observed in Experiment 1-increased sensitivity at the singleton location accompanied by a decrement in sensitivity at positions neighboring the singleton item. This suggests that the results obtained in Experiment 1 were not due to the fact that observers were making two discriminations, and it demonstrates that the mere presence of a singleton item was sufficient to elicit these attentional effects.

The inclusion of a true control condition (trials in which no singleton was present) provided a baseline for assessing facilitory and inhibitory effects. As suggested in Experiment 1, sensitivity at the singleton location is enhanced relative to this baseline, while sensitivity at adjacent locations falls below that of the control condition. In Experiment 1, sensitivity appeared to asymptote as the distance between the singleton and probe became larger; 

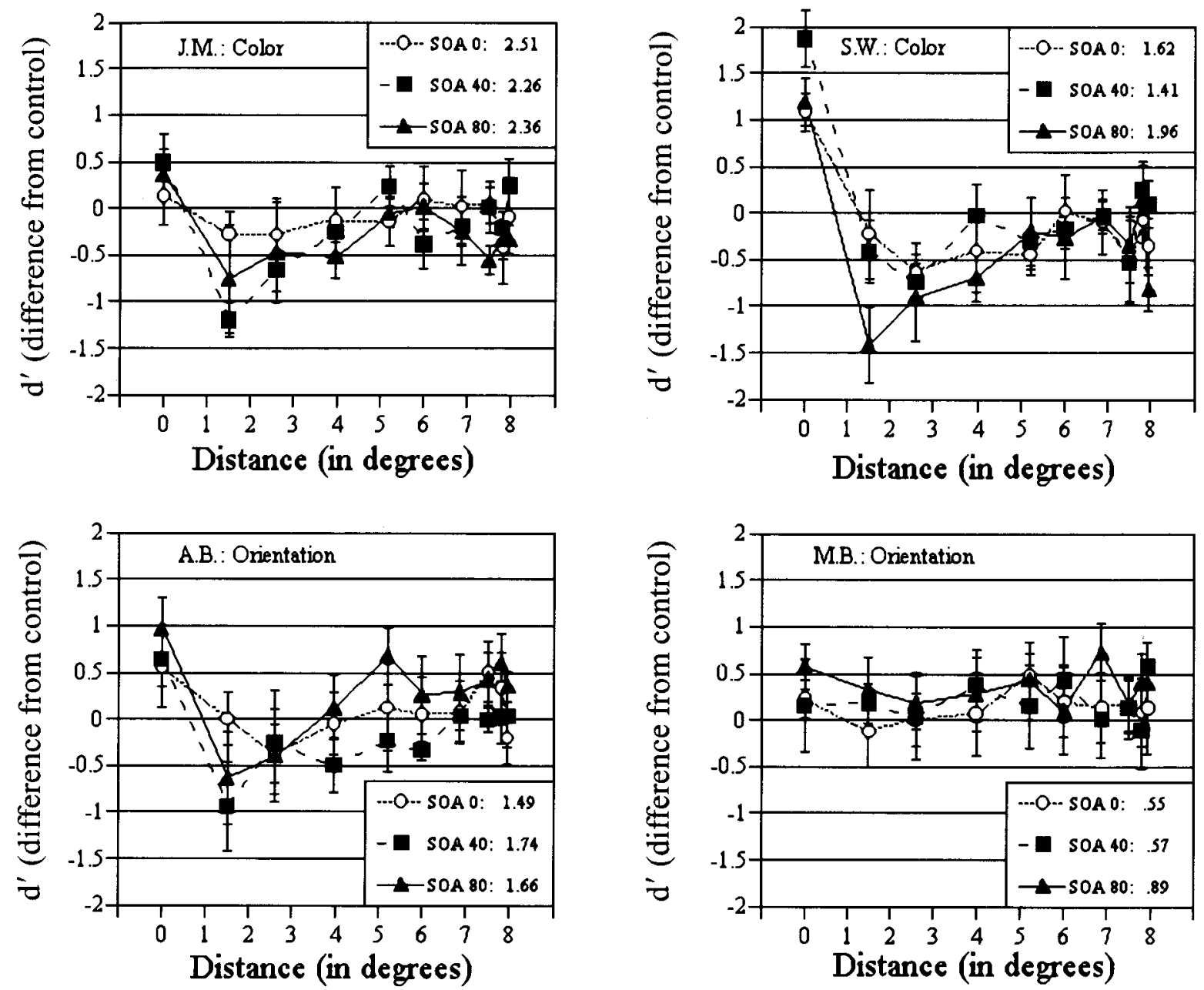

Figure 5. Form discrimination sensitivity $\left(d^{\prime}\right)$ relative to the control condition for each of the 4 observers in Experiment 2 . Sensitivity is plotted for each of the 10 singleton-probe separations and three stimulus onset asynchronies (SOAs). Error bars depict $\pm 1 S E$. Numbers following the SOA conditions in each legend represent the observer's sensitivity in the corresponding control condition.

the results of Experiment 2 reveal that this asymptote corresponds to control level sensitivity. In other words, once the separation between the probe and singleton reaches a certain distance, the singleton no longer influences probe sensitivity (see Caputo \& Guerra, 1998).

Finally, the predictions regarding the time course of attentional allocation were confirmed. Estimates for attentional allocation to an exogenous cue are in the range of 60-100 msec (Müller \& Rabbitt, 1989). Thus, in the SOA-0 condition, there may not be ample time for attention to be allocated to the singleton item. As a result, we would not expect to observe the pattern of facilitation and inhibition observed in Experiment 1. ${ }^{4}$ Note that this is true for Observers J.M., S.W., and A.B.-linear contrasts failed to detect a linear trend in the data, and a visual inspection of Figure 5 reveals that across the three SOAs, inhibition was at a minimum with SOA 0 . This finding is consistent with the hypothesis that the inhibitory effect is rooted in attentional processes.

\section{EXPERIMENT 3}

The results from Experiments 1 and 2 suggest that the allocation of attention - in this case to a unique stimulus feature-creates an inhibitory region surrounding the attended item. In the first two experiments, this inhibition appeared to extend out $4^{\circ}-5^{\circ}$ from the attended item. This suggests that there may be spatial limits to the extent of the inhibitory region, with selection mechanisms inhibiting elements within a specific spatial region surrounding the attended object. This is the type of prediction that ambiguity resolution theory would make because it is the size of the receptive fields in extrastriate areas that mandate the size of the inhibitory region. However, in Experiments 1 and 2, distance is perfectly confounded with the number of intervening elements; at the smallest probe-singleton distances, the probe is also directly adjacent to the singleton, and the number of intervening elements increases with distance. Thus, it is possible that 


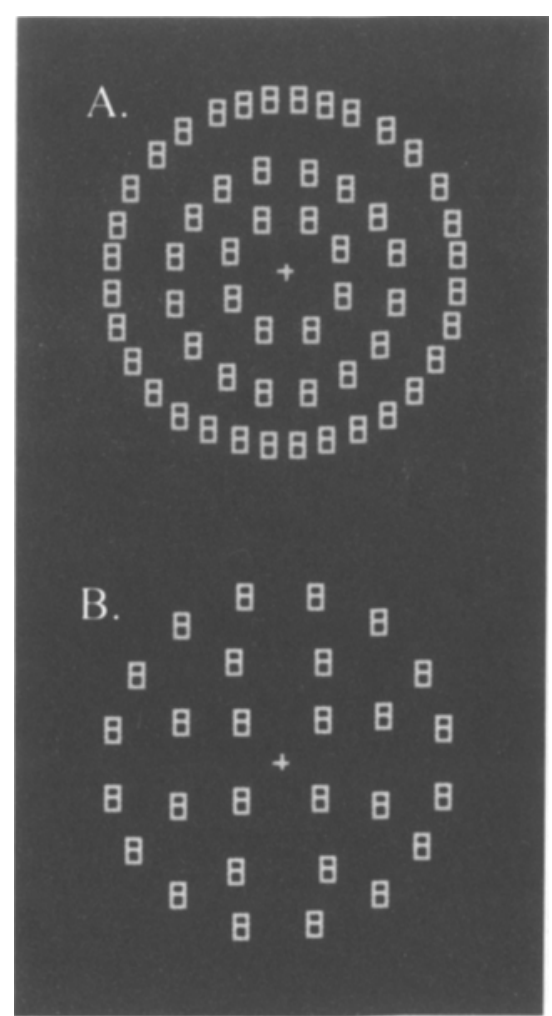

Figure 6. The stimulus array used in the dense (panel $A$ ) and sparse (panel B) conditions of Experiment 3. In both displays, the singleton and probe elements could appear at any of the locations in the middle ring of elements (16 locations in panel $A ; 8$ locations in panel B).

the strength of the inhibition is not spatially mediated, but instead is controlled by the number of intervening elements. Objects nearest to the attended object would receive strong inhibition, with the amount of inhibition decreasing as it spreads outward from object to object. According to this view, the strength of the inhibition would be a function the number of intervening items, rather than the actual spatial separation from the attended item.

Experiment 3 explores the nature of the inhibitory region by manipulating the density of the display elements. This will allow us to determine whether the inhibitory mechanisms operate on objects residing within a specific spatial region, or if the inhibition spreads outward to a certain number of objects. Experiment 3 also changes the spatial structure of the stimulus display to ensure that the results observed in Experiments 1 and 2 are not artifacts of the hexagonal grid used in those experiments.

\section{Method}

Observers and Apparatus. Three observers participated in this study; Observer S.W. also participated in Experiment 2. All 3 observers were paid for their participation. All observers reported normal color vision and normal or corrected-to-normal acuity. The apparatus was identical to that used in Experiments 1 and 2 .

Procedure. Stimulus elements were again block 8s. In this experiment, two different types of stimulus arrangements were used.
In both types of displays, the stimulus elements were arranged along three concentric circles with radii of $2^{\circ}, 4^{\circ}$, and $6^{\circ}$. In the dense stimulus display (shown in panel A of Figure 6), 8 elements were equally spaced along the inner ring, 16 elements formed the middle ring, and 32 elements were arrayed along the outer ring. The interelement spacing in this display closely matched that used in Experiments 1 and $2\left(1.54^{\circ}\right.$ between the centers of adjacent elements lying on the middle ring). In the sparse displays (illustrated in panel B of Figure 6), 4, 8, and 16 elements constituted the inner, middle, and outer rings, respectively. In both types of displays, singletons and probes always appeared in the middle ring, allowing for 16 possible singleton/probe locations in the dense display, and 8 possible locations in the sparse display. In the dense display, possible singleton-probe distances were $0^{\circ}, 1.54^{\circ}, 3.07^{\circ}, 4.43^{\circ}, 5.65^{\circ}$, $6.62^{\circ}, 7.38^{\circ}, 7.82^{\circ}$, and $8.00^{\circ}$. In the sparse condition, possible distances were $0^{\circ}, 3.07^{\circ}, 5.65^{\circ}, 7.38^{\circ}$, and $8.00^{\circ}$. Note that each of the singleton-probe distances in the sparse display has a corresponding distance in the dense display, allowing for a direct comparison across the two conditions.

For Observers S.W. and A.G., all elements were vertical, and the singleton differed in color. The value of the singleton (green or orange) alternated between sessions. For Observer M.S., all elements were green, and the singleton differed in terms of orientation $\left(0^{\circ}\right.$ or $22^{\circ}$ ). As in the first two experiments, the singleton display preceded the probe display; the singleton display was presented for $93 \mathrm{msec}$, followed by the probe display. In order to keep performance below ceiling, the duration of the probe display was set individually for each observer (S.W., $65 \mathrm{msec}$; A.G., $107 \mathrm{msec}$; M.S., $93 \mathrm{msec}$ ). For all observers, a poststimulus mask followed the target display. The mask consisted of 25 orange and green line segments randomly oriented and distributed around each of the stimulus locations.

During each experimental session, observers completed 544 experimental trials with the dense display and 576 experimental trials with the sparse display. Display type was blocked, and the order alternated between experimental sessions. Observers completed 25 practice trials before beginning each set of trials. In the set of 544 dense trials, the probes and singletons appeared equally often at each of the 16 possible locations on the middle ring of elements. Singletons were withheld on $1 / 17$ of the trials, creating a control condition, as in Experiment 2. Each type of probe (loss of top or bottom segment) appeared once for each combination of singleton and probe location. Across the 576 trials in the sparse condition, each type of probe appeared four times, with every possible combination of singleton and probe location across the eight possible stimulus locations (including the absence of a singleton on one out of nine of the trials).

Observers completed two practice and eight experimental sessions. Observers proceeded at their own pace, with a message appearing every 72 trials indicating that they should take a short rest break. Each session lasted approximately $1 \mathrm{~h}$.

\section{Results and Discussion}

For each singleton-probe distance in the dense and sparse conditions, the observer's sensitivity $\left(d^{\prime}\right)$ to make probe discrimination was measured. Figure 7 shows the 3 observers' sensitivities as a function of singleton-probe separation, for both the dense and the sparse displays. Again, across the 3 observers, we see a decrement in discrimination performance when the singleton and probe are in close spatial proximity, with the degree of decrement diminishing with distance.

ANOVAs were performed separately on the $d^{\prime}$ 's from the dense and sparse conditions for each observer (with 10 levels of distance for the dense condition and 6 levels for the sparse condition). These were followed by linear 

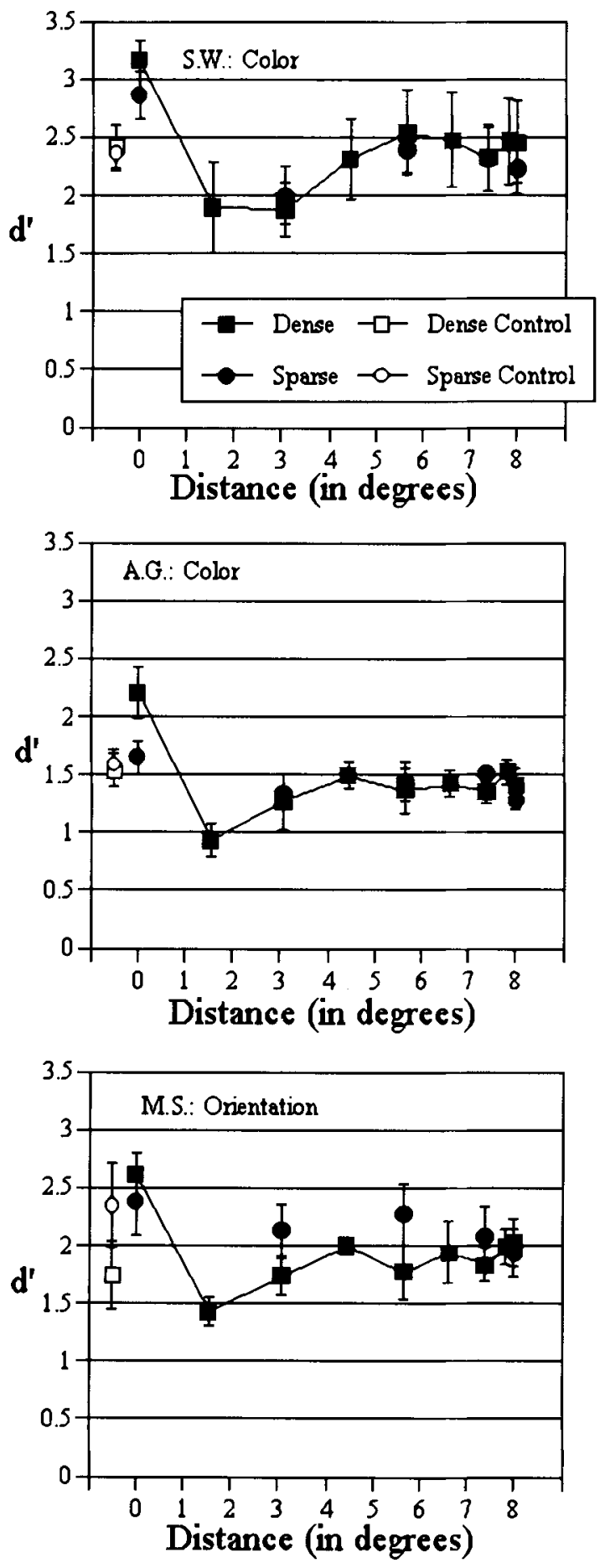

Figure 7. Form discrimination sensitivity $\left(d^{\prime}\right)$ for the 3 observers at each of the singleton-probe separations for both the dense and sparse conditions in Experiment 3. Performance in the dense conditions are denoted by filled squares; performance in the sparse conditions are denoted by filled circles. Open squares and open circles depict performance in the control conditions of the dense and sparse conditions, respectively. Error bars depict $\pm 1 S E$. contrasts similar to those performed in the prior experiments. For Observer S.W., the ANOVA on the dense condition yielded a main effect of distance $[F(9,63)=4.28$, $p \leq .001]$, as did the ANOVA for the sparse condition $[F(5,35)=5.43, p \leq .001]$. The linear contrast performed on the data from the dense condition revealed a reliable increase in sensitivity with distance $[F(1,7)=23.14, p \leq$ $.005]$, while the linear contrast for the sparse condition approached significance $[F(1,7)=4.76, p=.06]$. For Observer A.G., the main effect of distance was reliable in the dense condition $[F(9,63)=7.95, p \leq .001]$, but not in the sparse condition $[F(1,7)=1.99$, n.s. $]$. A linear contrast on the dense data revealed a significant linear trend $[F(1,7)=10.61, p \leq .05]$. Finally, for Observer M.S., the main effect of distance was reliable in the dense condition $[F(9,63)=4.97, p \leq .001]$, but not in the sparse condition $[F(5,35)=1.96$, n.s. $]$. A linear contrast confirms that the effect of distance in the dense condition is due at least in part to a linear trend in the data $[F(1,7)=13.96, p<.01]$.

In order to evaluate whether the density of the stimulus display affected discrimination performance, twotailed $t$ tests were performed on $d^{\prime}$ scores for each of the common singleton-probe distances. Specifically, the $t$ tests compared $d^{\prime}$ s from the dense and sparse conditions for each of the common singleton-probe distances: $0^{\mathbf{}}$, $3.07^{\circ}, 5.65^{\circ}, 7.38^{\circ}, 8.00^{\circ}$, and control. For Observers S.W. and M.S., none of the six $t$ tests approached statistical reliability. For Observer A.G., only the $t$ test for the $0^{\circ}$ separation reached the .05 significance level $[t(7)=$ $2.63, p \leq .05]$.

An examination of Figure 7 and the statistical analyses suggest that the inhibition affecting probe discriminability in the current task is mediated by spatial location. In other words, the amount of inhibition observed is a function of the distance from the attended object, rather than the number of intervening objects. This is particularly evident in S.W.'s and A.G.'s sensitivities for the separation of $3.07^{\circ}$. Even though the density of the objects differed greatly between the dense and sparse conditions, the sensitivities (and apparently the amount of inhibition) were virtually identical. This pattern was not as evident in the data of M.S.; however, note that Observers S.W. and A.G. performed with color singletons, while Observer M.S. performed with orientation singletons. Throughout this series of experiments, we have observed what appear to be weaker attentional effects resulting from the orientation singletons.

Finally, the level of performance in the two control conditions is of interest because it is an indication of the attentional salience of the probe. In essence, the dense/ sparse manipulation is one of set size, with 55 distractors in the dense display and 27 distractors in the sparse display. For Observers S.W. and A.G., there appeared to be no effect of set size, while a nonsignificant difference was present for Observer M.S. This suggests that the search for the probe was relatively efficient, at least in the absence of the singleton distractor. Experiment 4 examines 

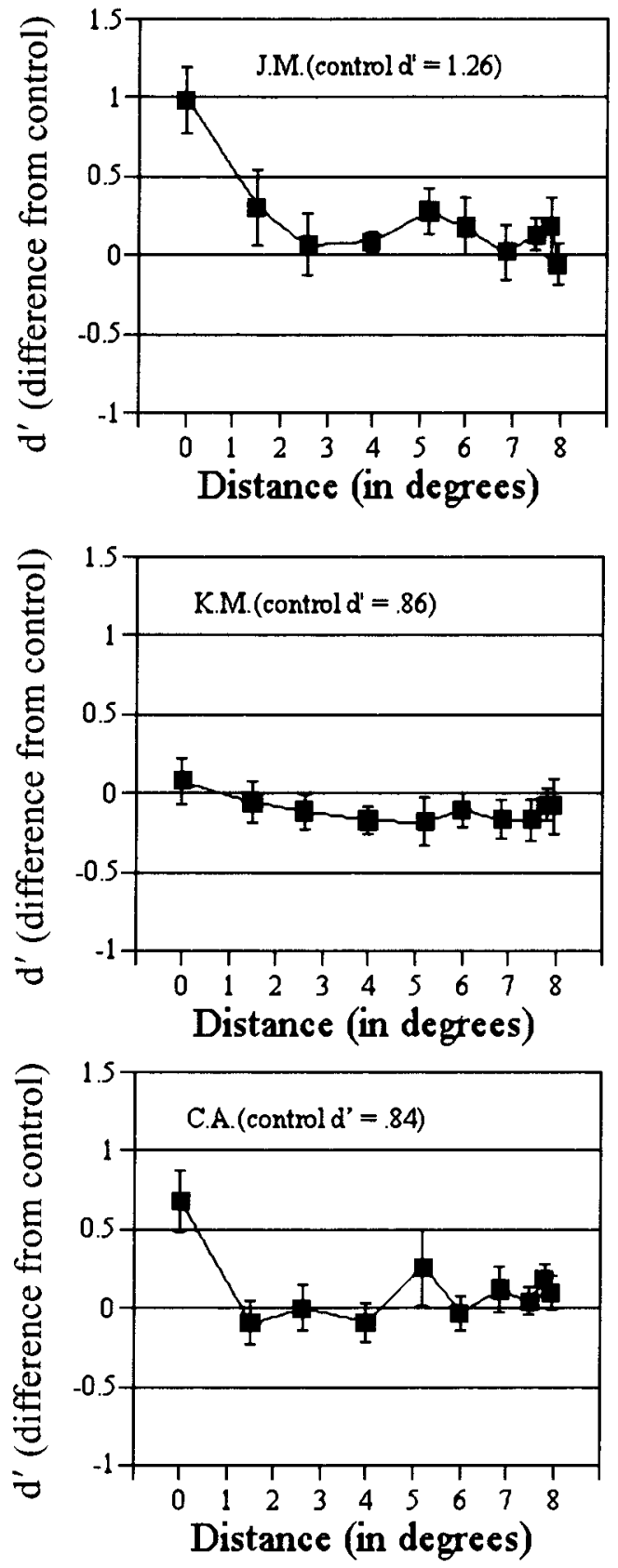

Figure 8. Probe detection sensitivity $\left(d^{\prime}\right)$ relative to the control condition for each of the 3 observers in Experiment 4. Sensitivity is plotted for each of the 10 singleton-probe separations. Error bars depict $\pm 1 S E$. Sensitivity in the control condition for each observer is displayed in the legend.

how the location of the singleton affects the detection of the probe.

\section{EXPERIMENT 4}

The task used in Experiments 1-3 can be conceptualized as one of detection followed by identification. Observers had to locate the form singleton probe among an array of distractors and then identify its form. The results observed in Experiments 1-3 could have arisen in several different ways. For example, the singleton distractor may have inhibited the processing of the probe at a relatively early level in the visual system, rendering the form singleton more difficult to detect (see, e.g., Cave \& Zimmerman, 1997; Hodgson, Müller, \& O'Leary, 1999). This implies that the probe identification decrements observed in Experiments 1-3 are actually rooted in difficulties in probe detection. Alternatively, if the inhibition occurs at a higher level in the visual system, probe detection would occur normally, while form identification processes would still be affected.

Experiment 4 evaluates these two alternatives by changing the task from one of probe identification to one of probe detection. If neighborhood inhibition affects processes relatively early in the visual system, we would expect to observe decrements in probe detection for locations surrounding an attentionally salient singleton distractor. On the other hand, if neighborhood inhibition has its effects later in the visual system, equivalent probe detection performance should be observed across the range of singleton-probe distances. Thus, the results of Experiment 4 should shed more light on the mechanisms underlying the localized inhibition currently under study.

\section{Method}

Observers. Three observers (including J.M.) participated in this study. The 2 observers other than J.M. were from the Geneseo Community and were paid for their participation. All observers reported normal color vision and normal or corrected-to-normal acuity.

Procedure. The apparatus and stimulus arrays were identical to those used in Experiments 1 and 2. A two-alternative forced-choice (2AFC) procedure was used, with the probe element present on only one of the intervals. When the probe was present, the SOA between array onset and the unmasking of the probe was $53 \mathrm{msec}$. For Observer K.M., the probe remained present for $130 \mathrm{msec}$; for $\mathrm{Ob}-$ server C.A., the probe display remained for $107 \mathrm{msec}$; and for Observer J.M., the probe display was presented for $93 \mathrm{msec}$. Distractor displays were identical in overall duration (e.g., $146 \mathrm{msec}$ total stimulus time for Observer J.M.). Stimulus displays within each interval were terminated by a mask (as in Experiments 2 and 3 ), presented for $507 \mathrm{msec}$, with $800 \mathrm{msec}$ elapsing between the offset of mask for Interval 1 and the onset of the stimulus array for Interval 2 .

Observers completed 25 practice and 624 experimental trials. On 312 of the trials, the probe was present in the first interval, and on the remainder of the trials, it was present in the second interval. Within the probe interval, the probe appeared equally often at each of the 12 possible locations, as did the singleton. The locations of the probe and singleton were uncorrelated across trials. In addition, the singleton was withheld on $1 / 13$ of the trials, providing a control condition. Within the distractor interval, the location of the singleton varied randomly among the 12 possible probe locations (and was also withheld on $1 / 13$ of the trials).

Observers completed two practice and six experimental sessions. All 3 observers performed with color singletons, and the color of the singleton (green among orange or vice versa) alternated between experimental sessions. Observers responded on the keyboard, indicating the interval that contained the probe. Errors were followed by a computer beep. Observers proceeded at their own pace, with a message appearing every 104 trials indicating that they should take a short rest break. Each session lasted approximately $45 \mathrm{~min}$. 


\section{Results and Discussion}

For each singleton-probe distance, the observer's probe detection sensitivity $\left(d^{\prime}\right)$ was measured-adjusting for the relative ease of the 2AFC task (Macmillan \& Creelman, 1991). Figure 8 shows the 3 observers' sensitivities (subtracted from their control condition performance) as a function of singleton-probe separation. ANOVAs were conducted on the $d^{\prime}$ data of each observer, with singleton-probe distance ( 11 levels including the control condition) treated as repeated measures. These ANOVAs were followed by linear contrasts, excluding the $0^{\circ}$ separation and control conditions. For Observer J.M., the initial ANOVA revealed a reliable effect of separation $[F(10,50)=4.09, p \leq .001]$. However, this was likely due to the enhanced performance for separation $0^{\circ}$, since the linear contrast was not reliable $[F(1,5)=2.36$, n.s. $]$. For Observer K.M., neither the initial ANOVA $[F(10,50)<$ 1] nor the subsequent contrast $[F(1,5)<1]$ was reliable. Finally, for Observer C.A., the initial ANOVA was reliable $[F(10,50)=3.17, p \leq .005]$, as was the subsequent linear contrast $[F(1,5)=7.20, p \leq .05]$.

As can be seen in Figure 8, there is little evidence for detection decrements at locations surrounding the singleton. Indeed, even for Observer C.A., for whom a reliable linear contrast effect obtained, sensitivity $\left(d^{\prime}\right)$ for probes adjacent to the singleton was only .09 below control level performance. The pattern of performance across the 3 observers suggests that the presence of color singletons did not inhibit the detection of the probes. This pattern obtained even though the detection of probes at the singleton location was facilitated for 2 of the observers, suggesting that attention was in fact drawn to the singleton location. These results suggest that, in Experiments 1-3, the form discrimination decrements observed near the singleton location probably are not due to detection difficulties, but rather suggest factors affecting the form identification process.

Using a dual-task procedure, Braun and Julesz (1998; see also Braun \& Sagi, 1990, 1991; Sagi \& Julesz, 1984) found that singleton detection performance is largely unaffected when attention is engaged by another stimulus in a display, whereas the identification of complex forms (e.g., letters) shows strong costs when attention is directed elsewhere. They argued that singleton detection is driven by saliency mechanisms operating early in visual processing, mechanisms that are independent of attention. Conversely, they argued that identification of complex forms occurs later in visual processing, relying on attentional mechanisms. As a result, performing their attentionally demanding second task detracts from letter identification performance, but not from singleton detection performance. Applying this logic to the present study, it suggests an attentional basis to the neighborhood inhibition under study. Probe detection, presumably relying on preattentive mechanisms, is unaffected by the direction of attention to a neighboring object, while probe identification, which presumably relies on attentive mechanisms, is inhibited in the neighborhood of an attentionally salient item.

\section{GENERAL DISCUSSION}

Multiple objects in a visual array pose potential problems for our visual systems. One solution to these problems is for our attentional system to inhibit the processing of unattended objects. Incorporating this idea, ambiguity resolution theory (Luck, Girelli, et al., 1997) proposes that only objects in the vicinity of the attended object need to be inhibited, a hypothesis termed neighborhood inhibition in the present study. According to this hypothesis, when visual attention selects an object, it does so in part by inhibiting the processing of objects near the selected object. Results from the present study, which used a probe discrimination task, support the neighborhood inhibition hypothesis. In Experiment 1, form discriminations of a probe presented near an attentionally salient and task-relevant singleton suffered in comparison to form discriminations of probes more distant from the singleton. Experiment 2 (through the inclusion of a true control condition) confirmed that the discriminations of neighboring items were inhibited relative to the control condition. Also, Experiment 2 demonstrated that the inhibitory region developed over time, consistent with the literature on the allocation of attention to exogenous cues (e.g., Cheal et al., 1994; Müller \& Rabbitt, 1989; Nakayama \& Mackeben, 1989). In Experiment 3, it was shown that this inhibitory region is rooted in the spatial distance from the attentionally salient object, rather than the number of intervening objects. In Experiment 4, the task was changed from probe discrimination to probe detection. The lack of an inhibitory region in Experiment 4 suggests that the probe discrimination decrements were not due to an inability to detect the probe items, but rather due to factors affecting form identification.

\section{Neighborhood Inhibition and Gradient Filters}

As described in the introduction, several recent studies have obtained results consistent with the present data, and therefore the neighborhood inhibition hypothesis. Specifically, these studies observed decrements in perceptual performance near the focus of attention (e.g., Bahcall \& Kowler, 1999; Caputo \& Guerra, 1998; Cave \& Zimmerman, 1997), suggestive of an inhibitory ring surrounding the attentional focus. The present study, as well as Caputo and Guerra and Cave and Zimmerman, found that attentional selection resulting from an exogenous cue (e.g., a color singleton) suppresses the processing of neighboring objects in the visual field. The data of Bahcall and Kowler show that such inhibitory effects also obtain when attention is directed via an endogenous cue. Moreover, these localized perceptual decrements have been observed with a variety of dependent measures: The present study employed a form discrimination task, Bahcall and Kowler measured letter identification accuracy, Cave and Zimmerman measured simple reaction time to a probe, and Caputo and Guerra measured line length thresholds.

One methodological constant across studies supporting neighborhood inhibition is that the attentionally selected 
object is always present concurrently with the object informing the response (i.e., the probe or target). For example, in the present study the singleton distractor remained present in the stimulus display after the introduction of the probe element. In Caputo and Guerra's (1998) study, the singleton distractor and the target item were presented simultaneously in a multi-item display. In Bahcall and Kowler's (1999) task, observers had to attend to two target letters presented simultaneously in a multiletter display. This methodological difference may explain the discrepancy between the studies that observed an inhibitory surround (Caputo \& Guerra, 1998; Cave \& Zimmerman, 1997) and those that observed a gradient structure to the attentional field (Cheal et al., 1994; Henderson \& Macquistan, 1993; LaBerge \& Brown, 1989; LaBerge et al., 1997). One possible explanation is that the removal of the attended object changes the spatial dynamics of the attentional field. With the attended object present, performance to the probe may reflect the consequences of attentional selection. By removing the attentionally selected object, probe performance may actually reflect the residue of attentional selection.

The present results are also difficult to reconcile with predictions of the various types of moving spotlight models of attention that are popular in the attention literature (e.g., Crick, 1984; Eriksen \& Yeh, 1985; Posner et al., 1980; Treisman \& Gelade, 1980). For example, Eriksen and Yeh's zoom lens model of attention proposes that attending to a location enables the further processing of items at that location, while further processing of items outside the focus of attention is inhibited. The present results are consistent with this prediction to a pointspecifically, the facilitation at the singleton location-but this model presumes a uniform inhibition of all unattended regions. As a consequence, it cannot explain the return to control levels of performance with increased distance from the attentional focus. Likewise, other spotlight models can explain the pattern of facilitation, but presume that performance away from the focus of attention should be uniformly low.

\section{An Alternative Explanation}

The data of Experiments 2 and 4 suggest an attentional component to the neighborhood inhibition hypothesis examined in this study. Nonetheless, an alternative, nonattentional explanation cannot be ruled out. It is possible that the feature singularity defining the singleton distractor alters the visual processing of neighboring items at a relatively early stage in the visual system (see, e.g., Knierim \& van Essen, 1992). Through local competitive interactions, items with unique features may gain in relative processing strength. Braun and Julesz (1998) have described such a model, in which the competition for visual salience is a preattentive process. The winners of this competition (i.e., the salient items) would then be made available to higher visual processes (or would at least be made available first). In this view, neighborhood inhibition actually results in attentional selection, rather than being caused by attentional selection. This explanation is consistent with the general pattern of neighborhood inhibition, particularly the data from the present Experiments 1 and 3. More assumptions need to be made, however, to account for the results of Experiments 2 and 4 . In Experiment 2, the inhibition was virtually absent at short SOAs, consistent with the buildup of attention to the singleton. From the preattentive competition explanation being offered, it is possible that larger lead times for the singleton (i.e., longer SOAs) provide a competitive advantage over the probe, resulting in the observed SOA effects. It is less clear why such competition early in the visual system would affect the discrimination, but not the detection of the probe, as was found in Experiment 4. Presumably, the ability to detect the probe depends on its salience, meaning that the probe's detectability is a gauge of its salience. According to this logic, the probe's salience (as measured by its detectability) does not appear to change as a function of the distance between the probe and the singleton. Thus, it is unclear why items with equivalent saliences should show differences in identification performance. Despite these potential inconsistencies, this nonattentional account of neighborhood inhibition remains a viable explanation of the data. Future research needs to address this possibility.

\section{Conclusion}

In conclusion, much recent research and theorizing has focused on the mechanisms of visual selective attention. As noted, one possible strategy for increasing the efficiency and accuracy of the processing of a selected object is to inhibit the processing of other objects in the visual field. However, as Luck, Girelli, et al. (1997) have pointed out, it may not be necessary to inhibit all objects in the visual field, but instead only those objects competing for representation among shared neural resources. This forms the basis of the neighborhood inhibition hypothesis, which is supported by the present study. These results, in concert with other recent work in the behavioral literature, parallel those obtained from physiological studies of visual selective attention. These data suggest that the attentional selection of an object, at least in part, occurs through the inhibition of neighboring objects.

\section{REFERENCES}

Allport, A. (1989). Visual attention. In M. I. Posner (Ed.), Foundations of cognitive science (pp. 631-682). Cambridge, MA: MIT Press. BACON, W. F., \& EGETH, H. E. (1994). Overriding stimulus-driven attentional capture. Perception \& Psychophysics, 55, 485-496.

BaHCALl, D. O., \& Kowler, E. (1999). Attentional interference at small spatial separations. Vision Research, 39, 71-86.

Braun, J., \& Julesz, B. (1998). Withdrawing attention at little or no cost: Detection and discrimination tasks. Perception \& Psychophysics, 60, 1-23.

Braun, J., \& SAGi, D. (1990). Vision outside the focus of attention. Perception \& Psychophysics, 48, 45-58.

Braun, J., \& SAGI, D. (1991). Texture-based tasks are little affected by a second task which requires peripheral or central attentive fixation. Perception, 20, 483-500.

Caputo, G., \& Guerra, S. (1998). Attentional selection by distractor suppression. Vision Research, 38, 669-689. 
Cave, K. R., \& Zimmerman, J. M. (1997). Flexibility in spatial attention before and after practice. Psychological Science, 8, 399-403.

Cepeda, N. J., Cave, K. R., Bichot, N. P., \& KiM, M.-S. (1998). Spatial selection via feature-driven inhibition of distractor locations. Perception \& Psychophysics, 60, 727-746.

Cheal, M. L., \& Lyon, D. R. (1992). Benefits from attention depend on the target type in location-precued discrimination. Acta Psychologica, 81, 243-267.

Cheal, M. L., Lyon, D. R., \& GotTlob, L. R. (1994). A framework for understanding the allocation of attention in location-precued discrimination. Quarterly Journal of Experimental Psychology, 47A, 699-739.

Chelazzi, L., Miller, E. K., Duncan, J., \& Desimone, R. (1993). A neural basis for visual search in inferior temporal cortex. Nature, 363, 345-347.

CRICK, F. H. C. (1984). Function of the thalamic reticular complex: The searchlight hypothesis. Proceedings of the National Academy of Sciences, 81, 4586-4590.

Desimone, R., \& Duncan, J. (1995). Neural mechanisms of selective attention. Annual Review of Neuroscience, 18, 193-222.

Desimone, R., Wessinger, M., Thomas, L., \& SChNeider, W. (1990). Attentional control of visual perception: Cortical and subcortical mechanisms. Cold Spring Harbor Symposium on Quantitative Biology, 55, 963-971.

DownING, C. J. (1988). Expectancy and visual-spatial attention: Effects on perceptual quality. Journal of Experimental Psychology: Human Perception \& Performance, 14, 188-202.

DunCAN, J. (1996). Cooperating brain systems in selective perception and action. In T. Inui \& J. L. McClelland (Eds.), Attention and performance XVI: Information integration in perception and communication (pp. 549-578). Cambridge, MA: MIT Press, Bradford Books.

ERIKSEN, C. W., \& Hoffman, J. E. (1972). Temporal and spatial characteristics of selective encoding from visual displays. Perception \& Psychophysics, 12, 201-204.

ERIKSEN, C. W., \& YEH, Y. Y. (1985). Allocation of attention in the visual field. Journal of Experimental Psychology: Human Perception \& Performance, 11, 583-597.

FolK, C. L., \& Remington, R. W. (1998). Selectivity in distraction by irrelevant featural singletons: Evidence for two forms of attentional capture. Journal of Experimental Psychology: Human Perception \& Performance, 24, 847-858.

Folk, C. L., Remington, R. W., \& Wright, J. H. (1994). The structure of attentional control: Contingent attentional capture by apparent motion, abrupt onset, and color. Journal of Experimental Psychology: Human Perception \& Performance, 20, 317-329.

Hawkins, H. L., Hillyard, S. A., Luck, S. J., Mouloua, M., DownING, C. J., \& WoODWARD, D. P. (1990). Visual attention modulates signal detectability. Journal of Experimental Psychology: Human Perception \& Performance, 16, 802-811.

Henderson, J. M., \& Macquistan, A. D. (1993). The spatial distribution of attention following an exogenous cue. Perception \& Psychophysics, 53, 221-230.

Hodgson, T. L., Müller, H. J., \& O’Leary, M. J. (1999). Attentional localization prior to simple and directed manual responses. Perception \& Psychophysics, 61, 308-321.

Houghton, G., \& Tipper, S. P. (1994). A model of inhibitory mechanisms in selective attention. In D. Dagenbach \& T. H. Carr (Eds.), Inhibitory processes in attention, memory, and language (pp. 53-112). San Diego: Academic Press.

JONIDES, J., \& YANTIS, S. (1988). Uniqueness of abrupt visual onset in capturing attention. Perception \& Psychophysics, 43, 346-354.

JosePH, J. S., \& OPTICAN, L. M. (1996). Involuntary attentional shifts due to orientation differences. Perception \& Psychophysics, 58, 651665.

KNiERIM, J. J., \& VAN ESSEN, D. C. (1992). Neuronal responses to static texture patterns in area V1 of the alert macaque monkey. Journal of Neurophysiology, 67, 961-980.

LABERGE, D., \& BROWN, V. (1989). Theory of attentional operations in shape identification. Psychological Review, 96, 101-124.
LaBerge, D., Carlson, R. L., Williams, J. K., \& Bunney, B. G. (1997). Shifting attention in visual space: Tests of moving-spotlight models versus an activity-distribution model. Journal of Experimental Psychology: Human Perception \& Performance, 23, 1380-1392.

Luck, S. J., Chelazzi, L., Hillyard, S. A., \& Desimone, R. (1997). Mechanisms of spatial selective attention in areas V1, V2 and V4 of macaque visual cortex. Journal of Neurophysiology, 77, 24-42.

LuCK, S. J., Girelli, M., McDermott, M. T., \& Ford, M. A. (1997). Bridging the gap between monkey neurophysiology and human perception: An ambiguity resolution theory of visual selective attention. Cognitive Psychology, 33, 64-87.

Macmillan, N. A., \& Creelman, C. D. (1991). Detection theory: A user's guide. New York: Cambridge University Press.

Miller, J. (1989). The control of visual attention by abrupt visual onsets and offsets. Perception \& Psychophysics, 45, 567-571.

Moran, J., \& Desimone, R. (1985). Selective attention gates visual processing in the extrastriate cortex. Science, 229, 782-784.

MotTER, B. C. (1993). Focal attention produces spatially selective processing in visual cortical areas V1, V2, and V4 in the presence of competing stimuli. Journal of Neurophysiology, 70, 909-919.

Müller, H. J., \& RABBiTT, P. M. A. (1989). Reflexive and voluntary orienting of visual attention: Time course of activation and resistance to interruption. Journal of Experimental Psychology: Human Perception \& Performance, 15, 315-330.

NAKAYAMA, K., \& MACKEBFN, M. (1989). Sustained and transient components of focal visual attention. Vision Research, 29, 1631-1647.

Posner, M. I., SNyder, C. R. R., \& Davidson, B. J. (1980). Attention and the detection of signals. Journal of Experimental Psychology: General, 109, 160-174.

Prinzmetal, W., Henderson, D., \& IvRy, R. B. (1995). Loosening the constraints on illusory conjunctions: The role of exposure duration and attention. Journal of Experimental Psychology: Human Perception \& Performance, 21, 1362-1375.

SAGI, D., \& JULESZ, B. (1984). Detection versus discrimination of visual orientation. Perception, 13, 619-628.

THEEUwes, J. (1991). Exogenous and endogenous control of attention: The effect of visual onsets and offsets. Perception \& Psychophysics, 49, 83-90.

Theeuwes, J. (1992). Perceptual selectivity for color and form. Perception \& Psychophysics, 51, 599-606.

TIPPER, S. P. (1985). The negative priming effect: Inhibitory priming by ignored objects. Quarterly Journal of Experimental Psychology, 37A, 571-590.

Treisman, A., \& Gelade, G. (1980). A feature-integration theory of attention. Cognitive Psychology, 12, 97-136.

Treisman, A., \& Gormican, S. (1988). Feature analysis in early vision: Evidence from search asymmetries. Psychological Review, 95, 1548.

TREISMAN, A., \& SCHMIDT, H. (1982). Illusory conjunctions in the perception of objects. Cognitive Psychology, 14, 107-141.

YANTIS, S., \& EGETH, H. E. (1999). On the distinction between visual salience and stimulus-driven attentional capture. Journal of Experimental Psychology: Human Perception \& Performance, 25, 661-676.

YANTIS, S., \& JoNIDES, J. (1984). Abrupt visual onsets and selective attention: Evidence from visual search. Journal of Experimental Psychology: Human Penception \& Performance, 10, 601-621.

\section{NOTES}

1. This probe was explicitly chosen to have a relatively low attentional salience. The probe was designated by abrupt offsets, which have been demonstrated to be weak attractors of attention (e.g., Jonides \& Yantis, 1988; Miller, 1989; Theeuwes, 1991; Yantis \& Jonides, 1984). However, the probe was the only element possessing an abrupt offset, in essence making it a feature singleton. Also, the removal of the line segment rendered the probe a form singleton (although targets defined by the lack of a feature have been shown to produce poor performance in search tasks, e.g., Treisman \& Gormican, 1988). Nonetheless, the fact that observers were able to reliably discriminate features of the 
probe suggests that its attentional salience was greater than that of the other "background" elements constituting the stimulus array.

2. In sessions in which a hit rate of 1.0 was obtained for one of the singleton-probe distances, it was replaced by $[1-(1 / 2 N)]$. Likewise, false alarm rates of 0 were replaced by $(1 / 2 N)$ (Macmillan \& Creelman, 1991).

3. The weights used in these linear contrasts were adjusted to reflect the unequal intervals among the singleton-probe separations. This adjustment was also made for the linear contrasts reported in the subsequent experiments.
4. The actual SOA values $(0,40$, and $80 \mathrm{msec})$ probably underestimate the time available for attention to be allocated to the singleton. The singleton remains after the presentation of the probe, and most likely has a lower attentional salience relative to the singleton. As a result, attention most likely continues to accrue to the singleton past the onset of the probe. This might explain why Observer J.M. showed stronger attentional effects with an SOA of 40 relative to $80 \mathrm{msec}$.

(Manuscript received September 11, 1998; revision accepted for publication July 28,1999 .) 